\title{
EXPLORANDO EVIDÊNCIAS CIENTÍFICAS SOBRE AÇÕES DE AUTOCUIDADO EM PACIENTES COM PSORÍASE: REVISÃO INTEGRATIVA
}

\section{EXPLORING SCIENTIFIC EVIDENCE ON SELF-CARE ACTIONS IN PATIENTS WITH PSORIASIS: AN INTEGRATIVE REVIEW}

\author{
Valéria Leite Soares ${ }^{1} *$ Márcia Queiroz de Carvalho Gomes ${ }^{2} *$ João Marçal Medeiros de Sousa $^{3}$ \\ Paula Soares Carvalho ${ }^{4}$ Esther Barros Palitot ${ }^{5} *$ Maria Júlia Guimarães Oliveira Soares ${ }^{6}$
}

\begin{abstract}
RESUMO
Objetivo: analisar artigos científicos publicados em periódicos online acerca das ações de autocuidado, desenvolvidas por profissionais de saúde, às pessoas com psoríase. Metodologia: Foram utilizadas buscas nas bases de dados durante o mês de agosto de 2020 - SCOPUS, LILACS, BDENF, CINAHL, Web Science e MEDLINE (via de acesso PubMed). Foram incluídas na busca publicações em inglês, espanhol e português do período de janeiro de 2015 a julho de 2020. Resultados: A amostra final foi composta por 9 artigos em inglês. A discussão do estudo tem como base teórica a Teoria do Déficit do Autocuidado de Dorothea Orem. Foram criadas 3 categorias a partir dos fundamentos e pressupostos que esta teoria oferece. Os resultados apontaram a educação em saúde como meio indispensável para aquisição de conhecimentos e habilidades para o autocuidado. Quanto as ações de autocuidado realizadas por profissionais de saúde, destacaram-se orientações e informações sobre conhecimentos - da doença e comorbidades, tratamento, medicação, mudanças no estilo de vida, hábitos saudáveis, manuseio das lesões cutâneas, outras. Fatores como aspectos emocionais, interações sociais, crenças, cultura, necessidades do paciente e motivação para autocuidar-se devem ser considerados. Considerações finais: $\mathrm{O}$ estudo possibilitou identificar e analisar evidências científicas acerca da temática. Constatou-se que para o desenvolvimento das ações de autocuidado é necessário identificar os requisitos de autocuidado do paciente, optar pela metodologia mais adequada e planejar as ações, podendo ou não seguir diretrizes padronizadas. A educação em saúde se coloca como essencial na comunicação dessas práticas, através de estratégias, utilizando-se de diferentes tecnologias em saúde.
\end{abstract}

Palavras-chave: Psoríase; Autocuidado; Doença Crônica; Profissional de Saúde

\begin{abstract}
Objective: to analyze scientific articles published in online journals about self-care actions, developed by health professionals, for people with psoriasis. Methodology: Searches were used in the databases during the month of August 2020 - SCOPUS, LILACS, BDENF, CINAHL, Web Science and MEDLINE (access via PubMed). Publications in English, Spanish and Portuguese from January 2015 to July 2020 were included in the search. Results: The final sample consisted of 9 articles in English. The discussion of the study is theoretically based on Dorothea Orem's Self-Care Deficit Theory. Three categories were created from the foundations and assumptions that this theory offers. The results indicated health education as an indispensable means for acquiring knowledge and skills for self-care. As for the selfcare actions performed by health professionals, guidelines and information on knowledge about the disease and comorbidities, treatment, medication, changes in lifestyle, healthy habits, handling of skin lesions, and others stood out. Factors such as emotional aspects, social interactions, beliefs, culture, patient needs and motivation to take care of themselves must be considered. Results: The final sample consisted of 9 articles in English. The discussion of the study is theoretically based on the Deficit Theory of the Final Considerations: The study made it possible to identify and analyze scientific evidence on the subject. It was found that for the development of self-care actions it is necessary to identify the patient's self-care requirements, choose the most appropriate methodology and plan the actions, which may or may not follow standardized guidelines. Health education is essential in the communication of these practices, through strategies, using different health technologies.
\end{abstract}

Keywords: Psoriasis; Self-care; Chronic Disease; Health Personnel

\footnotetext{
${ }^{1}$ Terapeuta Ocupacional. Doutoranda em Enfermagem UFPB. Docente adjunta do curso de Terapia Ocupacional UFPB. Membro do Grupo de Estudo e Pesquisa no Tratamento de Feridas (GEPEFE), João Pessoa, Brasil, ORCID: 0000-0002-6605-5907

${ }^{2}$ Terapeuta Ocupacional. Doutora. Docente Associada do curso de Terapia Ocupacional UFPB. João Pessoa, Brasil, ORCID: 00000002-4837-2968

${ }^{3}$ Graduando em Medicina UFPB. Diretor científico da Liga Acadêmica de Clínica Médica da UFPB (LACLIMED-UFPB). João Pessoa, Brasil, ORCID: 0000-0002-7299-1232

${ }^{4}$ Enfermeira. Mestranda em Enfermagem UFPB. Membro do Grupo de Estudo e Pesquisa no Tratamento de Feridas (GEPEFE). João Pessoa, Brasil, ORCID: 0000-0003-3680-6981

${ }^{5}$ Médica. Doutora. Membro do Group for Research and Assessment of Psoriasis and Psoriatic Arthritis (GRAPPA). Docente do curso de medicina UFPB. João Pessoa, Brasil, ORCID: 0000-0002-8195-2534

${ }^{6}$ Enfermeira. Docente titular do curso de Graduação em Enfermagem, Pós-Graduação (Mestrado e Doutorado) UFPB, líder do Grupo de Estudo e Pesquisa no Tratamento de Feridas (GEPEFE), João Pessoa, Brasil, ORCID: 0000-0001-8025-9802 https://doi.org/10.31011/reaid-2021-v.95-n.36-art.1201 Rev Enferm Atual In Derme v. 95, n. 36, 2021 e-021185 


\section{INTRODUÇÃO}

A psoríase é uma doença crônica não transmissível e recorrente, inflamatória e multissistêmica, com grande polimorfismo de expressão clínica e se manifesta de diferentes formas, sendo a mais comum (90\% dos casos), a forma vulgar. Caracterizada por hiperplasia epidérmica e ativação imune inapropriada, se manifesta na pele e articulações, produzindo lesões acompanhadas de prurido e dor. A doença apresenta importante predisposição genética, seu início e agravamento provém de fatores ambientais ou comportamentais. Hábitos de vida como álcool, tabagismo e estresse resultam em prejuízos significativos que favorecem o agravo da doença, pois ela apresenta ciclos de remissão e exacerbação ${ }^{(1-}$ 3).

Dificuldades e/ou incapacidades físicas e prejuízos psicossociais devido ao preconceito e estigma, são alguns danos acarretados pela psoríase. Concomitante a estes aspectos, existem as comorbidades tais como: hipertensão arterial sistêmica, obesidade; dislipidemia; depressão, enxaqueca, ansiedade, doença hepática gordurosa não alcoólica, diabetes mellitus, outras ${ }^{(4)}$.

A doença psoriásica ou psoríase não é incomum, sua prevalência no mundo varia entre 0.5 a $11.4 \%$, com maior incidência nos países frios. No Brasil, seu índice é de $1.3 \%$, porém apresenta variações entre as regiões. Nas regiões Norte, Nordeste e Centro-Oeste varia entre 0.9 a $1.1 \%$, enquanto que, nas regiões Sul e Sudeste a prevalência chega a 1.9\%. Acomete mais pessoas da raça branca e de diferentes idades, todavia, apresenta maior incidência entre 30 a 40 anos e 50 a 70 anos, tanto homens como mulheres ${ }^{(3)}$.

Devido à complexidade e seu longo curso, a psoríase prejudica as pessoas acometidas em diferentes áreas ocupacionais, seja pela limitação física, emocional ou social, ou mesmo, pela própria condição clínica da doença. Somado a essas condições, também há o alto custo do tratamento medicamentoso e os frequentes exames laboratoriais quando não se está em remissão ${ }^{(5)}$.

Diante da condição recorrente e remitente e da cronicidade, ações de autocuidado (AC) orientadas por profissionais de saúde às pessoas com psoríase, ganham destaque. Dorothea Orem define o AC como uma prática de atividades que os indivíduos iniciam e realizam em seu próprio benefício para manter a vida, a saúde e o bem-estar. Quando realizado com eficácia, busca promover alívio e minimização dos sinais e sintomas, previne agravos e abrange a reabilitação quando incapacidades temporárias ou definitivas se instalam ${ }^{(6)}$. A teórica formulou 3 categorias de requisitos de autocuidado que devem ser considerados para o planejamento das ações de autocuidado, estes estão descritos na Teoria do Déficit do Autocuidado - requisitos de autocuidado universais; requisitos de autocuidado de 
desenvolvimento e os requisitos de autocuidado no desvio de saúde ${ }^{(6-9)}$.

O objetivo desta revisão foi analisar artigos científicos publicados em periódicos online acerca das ações de autocuidado desenvolvidas por profissionais de saúde às pessoas com psoríase. Deste modo, entendese que esse estudo possa ser de relevância para o campo da saúde, ao se pretender levar a reflexão sobre a necessidade das práticas de autocuidado no contexto da psoríase, considerando a complexidade e cronicidade de uma doença, incapacitante e estigmatizante. Ao mesmo tempo, este estudo poderá subsidiar e/ou estimular novas pesquisas a acerca da temática, visto que, há poucos estudos abrangendo o autocuidado na doença psoriásica, principalmente publicações brasileiras.

\section{METODOLOGIA}

Esta revisão integrativa seguiu as seis etapas descritas por $\operatorname{Ganong}^{(10)} \quad$ - 1) estabelecimento da pergunta de pesquisa; 2) definição dos critérios de inclusão de estudos e seleção da amostra; 3) organização dos dados e categorização dos estudos, considerando as características em comum; 4) análise crítica dos achados, identificando diferenças e conflitos; 5) interpretação e discussão dos resultados e 6) apresentação da revisão.

Na elaboração da pergunta norteadora utilizou-se o acrônimo PIO, a forma mais básica. P - pessoas com psoríase; I - ações de autocuidado orientadas/realizadas por profissionais de saúde; $\mathrm{O}$ - a mitigação dos sinais e sintomas da psoríase; melhoria do bem-estar ${ }^{(11)}$. Desta forma, a pergunta central da pesquisa foi: "Quais as ações de autocuidado são oerientadas/realizadas por profissionais de saúde para mitigar os sinais e sintomas da psoríase e favorecer o bem-estar do paciente?

Estabeleceu-se como critérios de inclusão: artigos completos disponíveis eletronicamente, nos idiomas - português, inglês e espanhol publicados no período de janeiro de 2015 a julho de 2020, que apresentassem a temática proposta no título, ou no resumo ou nos descritores. Como critérios de exclusão: artigos de revisão, artigos que não atendessem a temática, resumos, opinião de especialistas, artigos em duplicidade, editoriais, conferências, carta ao editor, carta ao leitor e literatura cinzenta (teses, dissertações e monografias).

O levantamento bibliográfico foi realizado no mês de agosto de 2020 , por meio de busca eletrônica, através do portal de Periódicos Capes/MEC, acessando o sistema CAFe, acrônimo que corresponde a Comunidade Acadêmica Federada, caracterizado como um serviço de gestão de identidade, em que a Universidade Federal da Paraíba (UFPB) faz parte. Esse serviço permite às instituições dessa comunidade integrar suas bases de dados. 
Como estratégias de investigação, foram utilizados os Descritores em Ciências da Saúde (DeCS) utilizando o operador booleano AND: psoriasis AND self-care AND self-management, para as bases de dados SCOPUS, Literatura LatinoAmericana e do Caribe em Ciências da Saúde (LILACS), Base de dados em Enfermagem (BDENF), Cumulative Index to Nursing and Allied Health Literature (CINAHL). Na base Web Sciencie foram utilizados os mesmos descritores e o booleano AND, psoriasis AND self-care* e psoriasis AND self-management*. Para as bases de dados MEDLINE (via de acesso PubMed) os descritores do Medical Subject Headings $(\mathrm{MeSH})$ incluído o operador booleano OR: psoriasis AND self-care OR psoriasis AND self-management foram utilizados.
Os dados foram extraídos por um revisor, confirmados por um segundo revisor e as incongruências ou dúvidas foram resolvidas por consenso entre os autores.

Posteriormente, ao se definir quais informações seriam mais pertinentes para a organização dos dados, optou-se por construir dois quadros no Microsoft Word ${ }^{\circledR}$ (Quadro1 e Quadro 2). Dos dados emergiram 3 categorias para análise e discussão.

\section{RESULTADOS}

Na busca por descritores nas bases de dados foram achados 208 estudos. Após leitura dos títulos e palavras-chave, 105 artigos foram selecionados. Ao ler os resumos e utilizar os critérios de exclusão, 22 artigos foram selecionados para leitura na íntegra, destes, 09 foram incluídos no estudo, de acordo com o modelo PRISMA ${ }^{(12)}$ (Figura 1).

Figura 1 - Fluxograma do processo de identificação, seleção e inclusão dos estudos para compor a revisão integrativa. João Pessoa, PB, Brasil, 2021

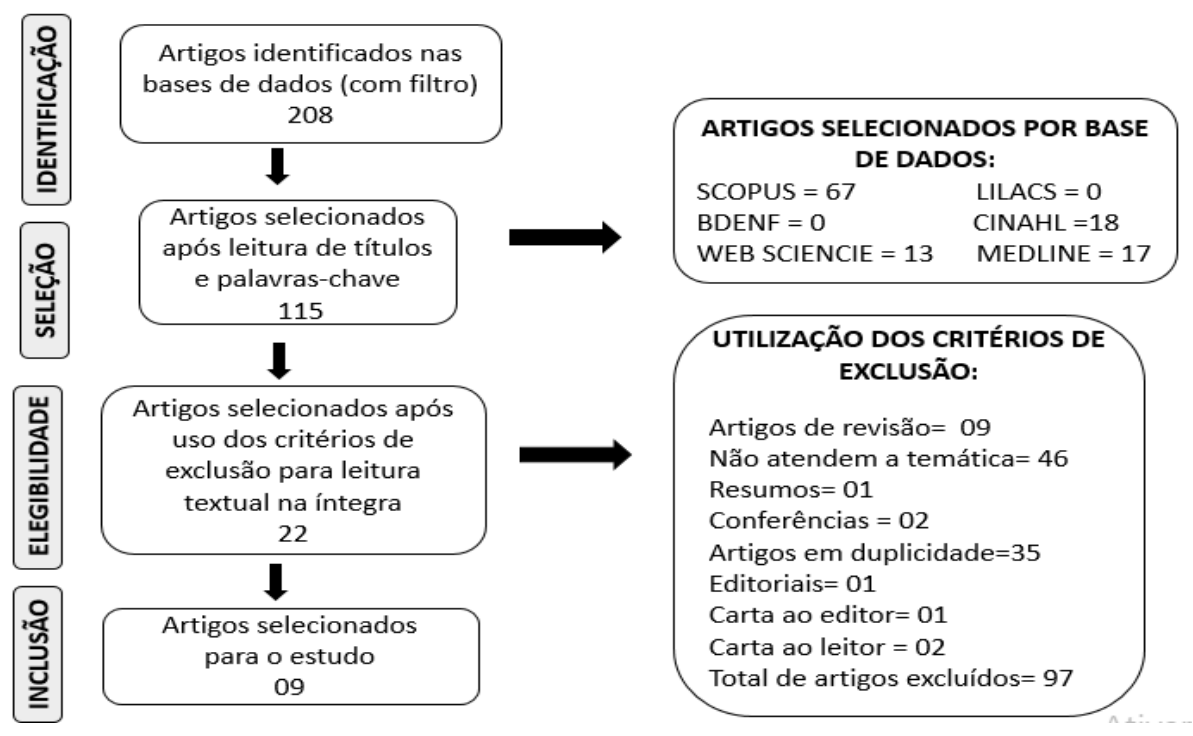

Fonte: Elaborado pelos autores, 2021 
Trata-se de um corpus composto em sua totalidade de literatura internacional, publicado no idioma inglês. Das publicações selecionadas, destacamos o ano de 2017 como o ano de maior volume de publicações $44,4 \%$ (4). Em 2019 com 33,3\% (3) publicações, em seguida, 2018 com 22,2\% (2). Não foram encontrados estudos dos anos de 2020, 2016 e 2015.

Os países que mais produziram artigos sobre a temática estudada pertencem ao continente europeu, totalizando 77,8\% (7) com destaque para o Reino Unido com 33,3\% (3) e Noruega e Dinamarca 22,2\% (2) cada. China e Irã publicaram $11,1 \%$ (1) artigo de cada país. Não foram encontradas publicações latino americanas. O maior quantitativo de publicações científicas desta temática nesses países, pode se justificar pela maior prevalência de casos, principalmente no continente europeu. Fatores genéticos e ambientais que envolvem a psoríase, são mais evidenciados em países de clima frio, quando comparados a países de clima tropical, com predomínio em caucasianos, enquanto fator $\operatorname{racial}^{(13)}$.

A maior concentração dos estudos foi encontrada na base de dados SCOPUS $88,9 \%$ (8), porém alguns se apresentavam em duplicidade em outras bases de dados. Somam-se a esses, 1 estudo na base de dados MEDLINE, correspondendo a 11,1\%.

Quadro 1 - Distribuição dos estudos incluídos na revisão integrativa de acordo com título, autores, procedência, ano, país e delineamento. João Pessoa, PB, Brasil, 2021.

\begin{tabular}{|c|c|c|c|}
\hline Título & AUTORES & $\begin{array}{c}\text { PROCEDÊNCIA/ } \\
\text { ANO/PAÍS }\end{array}$ & $\begin{array}{l}\text { DELINEAMENTO } \\
\text { (Tipo de pesquisa; } \mathrm{n} \text { de participantes; } \\
\text { intervenção) }\end{array}$ \\
\hline $\begin{array}{l}\text { E1. } \\
\text { Health literacy: } \\
\text { a new piece of } \\
\text { the puzzle in } \\
\text { psoriasis care? } \\
\text { A cross- } \\
\text { sectional study }\end{array}$ & $\begin{array}{l}\text { Larsen MH, } \\
\text { Strumse } \\
\text { YAS, Borge } \\
\text { CR, et al. }{ }^{(14)}\end{array}$ & $\begin{array}{l}\text { SCOPUS } \\
2019 \\
\text { Noruega }\end{array}$ & $\begin{array}{l}\text { Estudo transversal com } 825 \text { pacientes com } \\
\text { psoríase que realizaram terapia climática - } \\
\text { helioterapia entre } 2011 \text { - } 2016 \text {. } \\
\text { A ES como estratégia de autocuidado foi } \\
\text { avaliada pelo Health Literacy } \\
\text { Questionnaire (HLQ) }\end{array}$ \\
\hline $\begin{array}{l}\text { E2. } \\
\text { A Controlled } \\
\text { Study of the } \\
\text { Feasibility and } \\
\text { Efficacy of a } \\
\text { Cloud-Based } \\
\text { Interactive } \\
\text { Management } \\
\text { Program } \\
\text { Between } \\
\text { Patients with } \\
\text { Psoriasis and } \\
\text { Physicians. }\end{array}$ & $\begin{array}{l}\text { Zhu B, Wang } \\
\text { Y, Zhou X, et } \\
\text { al. }{ }^{(15)}\end{array}$ & $\begin{array}{l}\text { MEDLINE } \\
2019 \\
\text { China }\end{array}$ & $\begin{array}{l}\text { Estudo Piloto do tipo pesquisa intervenção } \\
\text { com } 79 \text { pacientes divididos aleatoriamente } \\
\text { em grupo controle }(\mathrm{n}=39) \text { e grupo } \\
\text { intervenção }(\mathrm{n}=40) \text {, durante } 12 \text { meses. } \\
\text { Os pacientes do grupo controle receberam } \\
\text { um manual de enfermagem sobre psoríase } \\
\text { e foram acompanhados regularmente. Os } \\
\text { pacientes do grupo intervenção foram } \\
\text { gerenciados por meio do aplicativo (app) } \\
\text { na plataforma em nuvem, com itens de } \\
\text { gerenciamento da psoríase iguais aos do } \\
\text { grupo controle. }\end{array}$ \\
\hline E3. & Larsen MH, & SCOPUS & Estudo transversal com a participação de \\
\hline
\end{tabular}




\begin{tabular}{|c|c|c|c|}
\hline $\begin{array}{l}\text { Associations } \\
\text { between disease } \\
\text { education, self- } \\
\text { management } \\
\text { support, and } \\
\text { health literacy } \\
\text { in psoriasis. }\end{array}$ & $\begin{array}{l}\text { Strumse YS, } \\
\text { Andersen } \\
\text { MH, et al. }{ }^{(16)}\end{array}$ & $\begin{array}{l}2019 \\
\text { Noruega }\end{array}$ & $\begin{array}{l}825 \text { pacientes adultos que participaram do } \\
\text { programa Norueguês de Terapia Hélio } \\
\text { Climática (THC) no período de } 2011 \text { - } \\
\text { 2017. Trata-se de um Programa } \\
\text { multidisciplinar de } 3 \text { semanas, oferecido } \\
\text { na Gran Canária que inclui terapia } \\
\text { climática, exercícios físicos, discussões em } \\
\text { grupo entre pares e ES abrangente. }\end{array}$ \\
\hline $\begin{array}{l}\text { E4. } \\
\text { Self-care for } \\
\text { older people } \\
\text { with psoriasis. }\end{array}$ & $\begin{array}{l}\text { Onselen J } \\
\text { Van. }{ }^{(17)}\end{array}$ & $\begin{array}{l}\text { SCOPUS } \\
2018 \\
\text { Reino Unido }\end{array}$ & $\begin{array}{l}\text { Estudo analítico descritivo sobre o } \\
\text { tratamento da psoríase relacionado ao } \\
\text { autocuidado em idosos com psoríase, } \\
\text { segundo critérios do National Institute For } \\
\text { Health And Care Excellence. }\end{array}$ \\
\hline $\begin{array}{l}\text { E5. } \\
\text { Experience of } \\
\text { Being Young } \\
\text { With Psoriasis: } \\
\text { Self- } \\
\text { Management } \\
\text { Support Needs. }\end{array}$ & $\begin{array}{l}\text { Rasmussen } \\
\text { GS, } \\
\text { Kragballe K, } \\
\text { Maindal HT, } \\
\text { et al. }{ }^{(18)}\end{array}$ & $\begin{array}{l}\text { SCOPUS } \\
2018 \\
\text { Dinamarca }\end{array}$ & $\begin{array}{l}\text { Estudo qualitativo, com análise } \\
\text { descritiva interpretativa, com } 21 \\
\text { jovens/adolescentes. A coleta de dados foi } \\
\text { realizada por meio de grupo focal e } \\
\text { entrevista semiestruturada com } 4 \\
\text { participantes para maior aprofundamento. }\end{array}$ \\
\hline $\begin{array}{l}\text { E6. } \\
\text { Facing the } \\
\text { dilemma of } \\
\text { patient-centred } \\
\text { psoriasis care: a } \\
\text { qualitative } \\
\text { study } \\
\text { identifying } \\
\text { patient needs in } \\
\text { dermatological } \\
\text { outpatient } \\
\text { clinics }\end{array}$ & $\begin{array}{l}\text { Khoury LR, } \\
\text { Skov L, } \\
\text { Møller T. }{ }^{(19)}\end{array}$ & $\begin{array}{l}\text { SCOPUS } \\
2017 \\
\text { Dinamarca }\end{array}$ & $\begin{array}{l}\text { Estudo qualitativo, com dados gerados a } \\
\text { partir de entrevista semiestruturada com } 16 \\
\text { pacientes com psoríase, em um } \\
\text { ambulatório de dermatologia hospitalar. }\end{array}$ \\
\hline $\begin{array}{l}\text { E7. } \\
\text { Identifying } \\
\text { training and } \\
\text { informational } \\
\text { components to } \\
\text { develop a } \\
\text { psoriasis self- } \\
\text { management } \\
\text { application. }\end{array}$ & $\begin{array}{l}\text { Safdari R, } \\
\text { Firoz A, } \\
\text { Masoorian } \\
\text { H. }{ }^{(20)}\end{array}$ & $\begin{array}{l}\text { SCOPUS } \\
2017 \\
\text { Irã }\end{array}$ & $\begin{array}{l}\text { Estudo descritivo-analítico realizado com } \\
100 \text { pacientes com psoríase e } 26 \\
\text { dermatologistas. } \\
\text { Foi aplicado um questionário para } \\
\text { pacientes e dermatologistas com o intuito } \\
\text { de identificar, quais são as informações } \\
\text { necessárias para um aplicativo, no } \\
\text { gerenciamento do autocuidado dos } \\
\text { pacientes com psoríase }\end{array}$ \\
\hline $\begin{array}{l}\text { E8. } \\
\text { 'New to me': } \\
\text { changing } \\
\text { patient } \\
\text { understanding } \\
\text { of psoriasis and } \\
\text { identifying } \\
\text { mechanisms of } \\
\text { change. The }\end{array}$ & $\begin{array}{l}\text { Nelson PA, } \\
\text { Kane K, } \\
\text { Pearce CJ, et } \\
\text { al. }^{(21)}\end{array}$ & $\begin{array}{l}\text { SCOPUS } \\
2017 \\
\text { Reino Unido }\end{array}$ & $\begin{array}{l}\text { Estudo quantitativo e qualitativo com } 55 \\
\text { pacientes com psoríase. Foram aplicados o } \\
\text { Questionário de Percepção de Doença } \\
\text { Revisada (IPQ-R modificado) e a Escala } \\
\text { Hospitalar de Ansiedade e Depressão } \\
\text { (HADS) em pacientes antes e após a } \\
\text { intervenção com material do Pso Well }{ }^{\circledR} \text {. } \\
\text { Escalas numéricas para avaliar as } \\
\text { percepções de mudança de entendimento }\end{array}$ \\
\hline
\end{tabular}




\begin{tabular}{|c|c|c|c|}
\hline $\begin{array}{l}\text { Pso Well® } \\
\text { patient } \\
\text { materials } \\
\text { mixed-methods } \\
\text { feasibility study }\end{array}$ & & & $\begin{array}{l}\text { sobre a psoríase e a ansiedade resultantes } \\
\text { do envolvimento com os materiais. } \\
\text { Entrevistas qualitativas foram realizadas } \\
\text { explorando a aceitabilidade e utilidade dos } \\
\text { materiais. }\end{array}$ \\
\hline $\begin{array}{l}\text { E9. } \\
\text { The role of } \\
\text { community } \\
\text { pharmacists in } \\
\text { supporting self- } \\
\text { management in } \\
\text { patients with } \\
\text { psoriasis. }\end{array}$ & $\begin{array}{l}\text { Tucker R, } \\
\text { Stewart D. }{ }^{(22)}\end{array}$ & $\begin{array}{l}\text { SCOPUS } \\
2017 \\
\text { Reino Unido }\end{array}$ & $\begin{array}{l}\text { Trata-se de um estudo piloto com análise } \\
\text { de pre e pós-intervenção. Sete farmácias } \\
\text { comunitárias foram selecionadas com base } \\
\text { em sua localização. Participaram } 7 \\
\text { farmacêuticos e } 47 \text { pacientes com psoríase } \\
\text { leve a moderada em tratamento tópico. } \\
\text { A intervenção foi realizada através de } 2 \\
\text { consultas farmacêuticas em um intervalo } \\
\text { de } 6 \text { semanas. } \\
\text { Foram aplicados a cada consulta o Person- } \\
\text { Centered Dermatology Self-Care Index } \\
\text { (PeDeSi) Self-Administrated Psoriasis } \\
\text { Area and Severity Index (SAPASI) e } \\
\text { Dermatology Life Quality Index (DLQI), } \\
\text { objetivando mensurar o conhecimento do } \\
\text { paciente acerca da doença, a sua } \\
\text { severidade e a qualidade de vida, } \\
\text { respectivamente. } \\
\text { As consultas constaram de aplicação dos } \\
\text { protocolos seguida de orientações verbais } \\
\text { e disponibilidade de links de acesso a } \\
\text { folhetos educativos online sobre a psoríase } \\
\text { e seu manejo. As orientações tinham como } \\
\text { base o escore do PeDeSi, o que permitia } \\
\text { identificar as necessidades de autocuidado } \\
\text { do paciente. }\end{array}$ \\
\hline
\end{tabular}

Fonte: Elaborado pelos autores, 2021

Quadro 2 - Distribuição dos estudos incluídos na revisão integrativa de acordo com autores, objetivo e desfecho. João Pessoa, PB, Brasil, 2021.

\begin{tabular}{|c|c|c|}
\hline AUTORES & OBJETIVO & DESFECHO \\
\hline $\begin{array}{l}\text { E1. } \\
\text { Larsen MH, } \\
\text { Strumse YAS, } \\
\text { Borge CR, et } \\
\text { al. }{ }^{(14)}\end{array}$ & $\begin{array}{l}\text { Investigar os pontos fortes e } \\
\text { fracos da educação em saúde } \\
\text { (ES) e suas dimensões em um } \\
\text { grupo de pacientes com } \\
\text { psoríase de moderada a grave; } \\
\text { verificar possíveis associações } \\
\text { entre a qualidade de vida, } \\
\text { características demográficas, } \\
\text { clínicas e de autocuidado. }\end{array}$ & $\begin{array}{l}\text { A ES permite que as pessoas desenvolvam seus } \\
\text { conhecimentos, habilidades e potencial para } \\
\text { mudanças de comportamentos. } \\
\text { Pacientes com comorbidades apresentam maior } \\
\text { dificuldade de vínculo com profissionais de } \\
\text { saúde, em compreender as informações e } \\
\text { conciliá-las aos procedimentos e } \\
\text { recomendações dos tratamentos, necessitando } \\
\text { assim, de maior suporte para o } \\
\text { autogerenciamento. } \\
\text { Constatou-se a importância do paciente saber } \\
\text { transitar/usar o sistema de saúde e o suporte } \\
\text { social para o autogerenciamento da própria } \\
\text { saúde. }\end{array}$ \\
\hline
\end{tabular}




\begin{tabular}{|c|c|c|}
\hline $\begin{array}{l}\text { E2. } \\
\text { Zhu B, Wang } \\
\text { Y, Zhou X, et } \\
\text { al. }^{(15)}\end{array}$ & $\begin{array}{l}\text { Investigar a viabilidade e } \\
\text { eficácia do gerenciamento } \\
\text { interativo da psoríase entre } \\
\text { paciente, médico e enfermeiro } \\
\text { usando um aplicativo } \\
\text { multimídia baseado em } \\
\text { nuvem. }\end{array}$ & $\begin{array}{l}\text { Para pacientes com psoríase, o uso da } \\
\text { plataforma interativa baseada em nuvem, } \\
\text { permitiu que os pacientes controlassem sua } \\
\text { doença de forma eficaz, melhorando a } \\
\text { comunicação com seus médicos e enfermeiros. } \\
\text { A interação em nuvem trouxe melhor } \\
\text { compreensão do tratamento, melhoria na saúde } \\
\text { mental e na qualidade de vida para os } \\
\text { pacientes, permitindo o controle e } \\
\text { gerenciamento da doença por eles, com mais } \\
\text { eficiência. }\end{array}$ \\
\hline $\begin{array}{l}\text { E3. } \\
\text { Larsen MH, } \\
\text { Strumse YS, } \\
\text { Andersen MH, } \\
\text { et al. }{ }^{(16)}\end{array}$ & $\begin{array}{l}\text { Investigar possíveis } \\
\text { associações entre ES } \\
\text { abrangente e ES específica } \\
\text { para as questões da psoríase; } \\
\text { Examinar as fontes essenciais } \\
\text { de informações sobre a doença } \\
\text { e como elas são avaliadas. }\end{array}$ & $\begin{array}{l}\text { A ES voltada para psoríase, se mostrou mais } \\
\text { promissora em relação a ES recebida de forma } \\
\text { abrangente. Pacientes que frequentaram o } \\
\text { programa mais de uma vez, apresentaram } \\
\text { maiores escores em ES e maior habilidade para } \\
\text { o autocuidado com melhor eficácia. } \\
\text { As fontes de informações mais importantes } \\
\text { sobre a psoríase foram os dermatologistas e os } \\
\text { amigos. }\end{array}$ \\
\hline $\begin{array}{l}\text { E4. } \\
\text { Onselen J } \\
\text { Van. }{ }^{(17)}\end{array}$ & $\begin{array}{l}\text { Apresentar informações } \\
\text { práticas sobre como avaliar a } \\
\text { psoríase na pessoa idosa e } \\
\text { ajudar a este grupo de } \\
\text { pacientes a autogerir a doença. }\end{array}$ & $\begin{array}{l}\text { O estudo apontou alguns princípios sobre os } \\
\text { cuidados necessários à pessoa idosa com } \\
\text { psoríase. Enfatizou a necessidade de suporte e } \\
\text { informações ao paciente e familiares sobre o } \\
\text { diagnóstico da doença e as opções de } \\
\text { tratamento; os fatores de risco e de estilo de } \\
\text { vida; como usar tratamentos prescritos com } \\
\text { segurança e eficácia; quando e como buscar } \\
\text { uma revisão clínica mais ampliada ou } \\
\text { especializada; discutir estratégias para lidar } \\
\text { com o impacto em seu bem-estar físico, } \\
\text { psicológico e social. }\end{array}$ \\
\hline $\begin{array}{l}\text { E5. Rasmussen } \\
\text { GS, Kragballe } \\
\text { K, Maindal HT, } \\
\text { et al. }{ }^{(18)}\end{array}$ & $\begin{array}{l}\text { Compreender as perspectivas } \\
\text { dos jovens/adolescentes em } \\
\text { relação a vida diária e suas } \\
\text { necessidades de suporte de } \\
\text { autocuidado. }\end{array}$ & $\begin{array}{l}\text { Identificou-se a necessidade do profissional de } \\
\text { saúde entender a adolescência quanto aos } \\
\text { aspectos emocionais e sociais e autoimagem, } \\
\text { característicos dessa fase para melhor } \\
\text { orientação das práticas de autocuidado. } \\
\text { Observou-se que a falta de apoio emocional e } \\
\text { social, a falta de relação de confiança e de } \\
\text { continuidade do tratamento com os mesmos } \\
\text { profissionais de saúde, comprometem a } \\
\text { aquisição de conhecimentos e as habilidades } \\
\text { necessárias para saber lidar com sua condição } \\
\text { de saúde e consequentemente, para o } \\
\text { autocuidado. }\end{array}$ \\
\hline $\begin{array}{l}\text { E6. } \\
\text { Khoury LR, } \\
\text { Skov L, Møller } \\
\text { T. }^{(19)}\end{array}$ & $\begin{array}{l}\text { Explorar as necessidades não } \\
\text { satisfeitas e as percepções de } \\
\text { saúde das pessoas com } \\
\text { psoríase quanto à interação } \\
\text { com os médicos e a estrutura } \\
\text { inerente às consultas no }\end{array}$ & $\begin{array}{l}\text { O estudo revelou ser fundamental a escuta das } \\
\text { necessidades do paciente, no que ele considera } \\
\text { importante para o seu cuidado. } \\
\text { Também se faz necessário atenção aos fatores } \\
\text { emocionais e preocupações do paciente, pois } \\
\text { sentimentos de estigmatização e mal }\end{array}$ \\
\hline
\end{tabular}




\begin{tabular}{|c|c|c|}
\hline & ambulatório de dermatologia. & $\begin{array}{l}\text { entendimento sobre a doença afetam as } \\
\text { estratégias de autogerenciamento do cuidado. } \\
\text { O apoio e a supervisão do profissional de saúde } \\
\text { para o encorajamento de comportamentos e } \\
\text { hábitos saudáveis, assim como, para as } \\
\text { intervenções de autocuidado, podem ser } \\
\text { realizadas de forma verbal e/ou com a } \\
\text { utilização de material ilustrativo e escrito. } \\
\text { Identificou-se a necessidade de ações de ES } \\
\text { individualizadas para esclarecer conceitos } \\
\text { errôneos sobre a doença e } \\
\text { apoiar comportamentos para a promoção da } \\
\text { saúde. }\end{array}$ \\
\hline $\begin{array}{l}\text { E7. } \\
\text { Safdari R, Firoz } \\
\text { A, Masoorian } \\
\text { H. }{ }^{(20)}\end{array}$ & $\begin{array}{l}\text { Identificar componentes } \\
\text { informativos e de treinamento } \\
\text { em autocuidado em psoríase } \\
\text { para o desenvolvimento de um } \\
\text { aplicativo para } \\
\text { autogerenciamento da } \\
\text { psoríase. }\end{array}$ & $\begin{array}{l}\text { Os dermatologistas e pacientes consideram } \\
\text { importante informar e treinar os pacientes para } \\
\text { práticas de autocuidado sobre: } \\
\text { - As principais manifestações e comorbidades } \\
\text { da doença e seus diversos tratamentos } \\
\text { (farmacológicos e não farmacológicos); } \\
\text { - O gerenciamento de estilo de vida } \\
\text { (nutricional, do estresse e de atividade física, } \\
\text { incluindo técnicas de relaxamento); } \\
\text { - Rastreamento de sintomas de depressão; } \\
\text { - Nutrição adequada e consumo de vitaminas e } \\
\text { suplementos. } \\
\text { Os componentes acima citados foram } \\
\text { considerados elegíveis para a criação de um } \\
\text { aplicativo eficaz para o autocuidado em } \\
\text { pacientes com psoríase. }\end{array}$ \\
\hline $\begin{array}{l}\text { E8. } \\
\text { Nelson PA, } \\
\text { Kane K, Pearce } \\
\text { CJ, et al. }{ }^{(21)}\end{array}$ & $\begin{array}{l}\text { Investigar se os materiais Pso } \\
\text { Well® ampliam a } \\
\text { compreensão da psoríase sem } \\
\text { aumentar a ansiedade; se são } \\
\text { bem aceitos; e se produzem } \\
\text { mudanças. }\end{array}$ & $\begin{array}{l}\text { O estudo apontou que o novo material do Pso } \\
\text { Well@ teve boa aceitação por parte dos } \\
\text { pacientes e apresentou boa compreensão, } \\
\text { coerência e ponderações para o controle da } \\
\text { psoríase, sem aumentar a ansiedade diante das } \\
\text { informações. }\end{array}$ \\
\hline $\begin{array}{l}\text { E9. Tucker R, } \\
\text { Stewart D. }{ }^{(22)}\end{array}$ & $\begin{array}{l}\text { Avaliar o impacto de uma } \\
\text { intervenção educacional } \\
\text { realizada por farmacêuticos } \\
\text { comunitários para melhorar o } \\
\text { autogerenciamento de } \\
\text { pacientes com psoríase. }\end{array}$ & $\begin{array}{l}\text { O apoio assistido por farmacêuticos a pacientes } \\
\text { com psoríase melhorou o conhecimento sobre a } \\
\text { psoríase e seu manejo, reduziu a gravidade da } \\
\text { doença e o impacto na qualidade de vida, } \\
\text { facilitando o autocuidado eficaz. }\end{array}$ \\
\hline
\end{tabular}

Fonte: Elaborado pelos autores, 2021

\section{DISCUSSÃO}

As ações de autocuidado são deliberadas pelos próprios sujeitos com a intenção de prover e/ou manter a integridade humana e suas habilidades, são impulsionadas pela educação em saúde e condicionadas por vários fatores a saber: estado de saúde, fase de desenvolvimento, idade, sexo, condições ambientais e fatores socioculturais. Hábitos e comportamentos podem ser adquiridos, modificados ou mesmo mantidos quando novos conhecimentos vão se somando aos conhecimentos prévios, favorecendo a promoção, restauração e manutenção da saúde 
e bem-estar, e se tratando da psoríase, somase a atenção para a prevenção e o cuidado das comorbidades com outras doenças crônicas $^{(6)}$.

As demandas de autocuidado terapêutico buscam atender os requisitos de autocuidado, entendidos como objetivos formulados para o desenvolvimento de suas ações. Diferentes meios podem ser usados para alcançar tais objetivos, visando atender as necessidades do paciente. Neste sentido, deve-se identificar quais são os requisitos de autocuidado do paciente, escolher o método pelo qual cada requisito identificado pode ser atendido e então, planejar as ações a serem realizadas $^{(6)}$.

São 3 os requisitos de autocuidado universais, de desenvolvimento e do desvio da saúde. Tomado como base estes requisitos, 3 categorias foram criadas para a discussão desta revisão, entendendo que as ações de autocuidado identificadas nos estudos selecionados podem estar agrupadas em mais de um dos três requisitos, pois para Orem ${ }^{(6)}$, eles se inter-relacionam.

\section{Demanda de autocuidado na perspectiva} dos requisitos de autocuidado universais

Vinculados aos processos de vida e pertinentes a todas as fases de desenvolvimento, os requisitos de autocuidado universais são os que mantém a integridade da estrutura e do funcionamento humano, enquanto organismo ${ }^{(6)}$. Estes requisitos estão relacionados com a nossa https://doi.org/10.31011/reaid-2021-v.95-n.36-art.1201 Rev Enferm Atual In Derme v. 95, n. 36, 2021 e-021185 sobrevivência e manutenção da saúde. São referentes a ingestão de líquidos, excreção, alimentação (tanto em quantidade como qualidade), respiração, a execução de atividades e descanso, interação e isolamento social, dentre outros requisitos que favorecem a prevenção de riscos à vida, à saúde e a promoção da saúde. Destarte, estes estão vinculados às atividades de vida diária, trabalho, lazer, atividades físicas, outras ${ }^{(7-9)}$.

Considerando a complexidade da psoríase e sua cronicidade, hábitos e comportamentos relacionados às atividades $\mathrm{e}$ exercícios físicos e de relaxamento, dieta alimentar e aspectos nutricionais e atividades do cotidiano são essenciais para a manutenção e promoção da saúde ${ }^{(15,20,21)}$. As práticas de autocuidado devem considerar também, as dimensões psicossociais relacionadas ao manejo do estresse e o gerenciamento da ansiedade e da depressão. Tais dimensões estão relacionadas com a atividade e repouso, e a participação social do paciente tais como o lazer, trabalho, vida sexual, atividades sociais, entre outras ${ }^{(17-21)}$.

Além da própria doença, as comorbidades ligadas à psoríase, classificadas em clássicas, emergentes, relacionadas ao estilo de vida e decorrentes do tratamento, exigem o envolvimento do paciente e dos profissionais de saúde, buscando a integralidade do cuidado em uma abordagem terapêutica longitudinal ${ }^{(23,24)}$. Neste contexto, a diabetes, doenças cardiovasculares, doenças gastrointestinais, obesidade, síndrome 10 
metabólicas, artrite psoriásica, depressão e outras comorbidades exigem cuidados com os aspectos físicos, psicológicos e sociais. Evitar o sedentarismo, se apropriar de hábitos alimentares saudáveis, sono regular, equilíbrio emocional, participação social evitando comportamentos de risco (tabagismo, álcool, alimentos impróprios, etc.), são necessários para a manutenção da saúde e prevenção de agravos e quadros $\operatorname{agudos}^{(15,17-19,21)}$.

Os achados de Melo et al. ${ }^{(25)}$ apontam que o consumo de alimentos inapropriados em substituição de frutas, legumes e outros alimentos, além do sedentarismo ligado a inatividade física, são alguns dos fatores de risco para as doenças crônicas. Cunha et al. ${ }^{(26)}$ também corroboram quando estudam sobre o autocuidado do paciente com infarto do miocárdio, afirmando que o estilo de vida sedentário, relacionado ao pouco interesse pela atividade física, pode ser identificado como requisito universal na manutenção do equilíbrio entre atividade e repouso. Os autores relatam que a ingesta de água, alimentação adequada, concomitante com atividade física, influenciarão na boa disposição física e respiratória, hidratação, excreção do organismo, todos identificados como requisitos universais, que quando não atendidos são considerados fatores de risco.

\section{Demandas de autocuidado na perspectiva} dos requisitos de autocuidado do desenvolvimento https://doi.org/10.31011/reaid-2021-v.95-n.36-art.1201 Rev Enferm Atual In Derme v. 95, n. 36, 2021 e-021185
Estes requisitos estão associados aos processos de desenvolvimento e as condições, e eventos que ocorrem durante os vários estágios do ciclo de vida (infância, adolescência, adultez e velhice). São requisitos que podem afetar de forma adversa o desenvolvimento ${ }^{(6)}$. É importante considerar a fase de desenvolvimento em que se encontra o paciente, pois cada fase vai apresentar necessidades distintas diante das diferentes condições de autonomia, cognitiva, sociais e emocionais, próprias de cada fase.

A psoríase pode ocorrer em qualquer idade, atingindo diferentes fases do desenvolvimento, mas apresenta maior pico em dois momentos da adultez ${ }^{(3)}$. Destarte, afetam a transição de fases da adultez, entre o adulto jovem para o adulto médio e entre o adulto médio para o adulto velho, interferindo em diferentes atividades e papéis característicos de cada fase ${ }^{(27)}$. O primeiro momento compromete idades (30 - 40 anos) de maior desenvolvimento produtivo e econômico, afetando as atividades de trabalho, participação social, atividade sexual e reprodutiva, relações afetivas responsabilidades familiares e financeira. $\mathrm{O}$ segundo momento (50 - 70 anos) compromete as atividades citadas anteriormente, com menor impacto na questão reprodutiva, porém afeta o adulto velho, com características próprias desta fase $\mathrm{f}^{(3,27)}$.

Conforme a fase de desenvolvimento em que o paciente se encontra, diferentes fatores devem ser considerados, aspectos 11 
físicos, psicológicos, sociais, culturais e ocupacionais. Considerando os modos como vivem, as perspectivas em relação à vida, a doença e ao tratamento, são fatores considerados importantes quanto às práticas de autocuidado e adesão ao tratamento. Cada fase do desenvolvimento pode apresentar potencialidades e fragilidades para a execução do autocuidado, podendo este ser executado com eficácia ou desencadear déficits para sua realização.

A maioria dos estudos selecionados não citam a fase de desenvolvimento de forma específica, mas descrevem sobre a influência da psoríase em diversos aspectos da vida comuns da adultez. Apenas um estudo coloca a psoríase como uma doença que pode ocorrer em qualquer idade ${ }^{(20)}$. Dois estudos abordam duas fases específicas do desenvolvimento, a adolescência e a velhice ${ }^{(17,18)}$. Não foram encontrados estudos que abordassem o autocuidado em psoríase na infância e nem na gestação, evento que pode ocorrer na adolescência e adultez.

A adolescência é uma fase de transição entre a infância e a adultez em que o adolescente direciona o foco de suas relações para o seu grupo, e não mais para a família. É um período em que ocorrem diversas mudanças físicas, psicológicas, comportamentais e sociais. Muitas vezes, os adolescentes se sentem desajeitados, se preocupam com sua aparência física, valorizando a estética corporal. Os aspectos relacionados ao seu desenvolvimento e https://doi.org/10.31011/reaid-2021-v.95-n.36-art.1201 Rev Enferm Atual In Derme v. 95, n. 36, 2021 e-021185 crescimento são alvos de frequente comparações, gerando preocupação de ser diferente e não ser aceito. É através da identificação e comparação com outros adolescentes, que eles começam a ter ideia de sua nova identidade corporal, podendo parecer-lhes anormais ou diferentes em relação aos seus pares ${ }^{(28)}$. $\mathrm{O}$ apoio ao adolescente com doenças crônicas precisa ter seu foco nos aspectos psicossociais, com atenção às características peculiares desta fase, devido ao valor que eles atribuem a aparência e ao corpo ${ }^{(18)}$.

Nas práticas do autocuidado, os profissionais de saúde acabam se preocupando mais com as questões clínicas da doença, buscam oferecer conhecimento e manejo sobre a psoríase, sem dar a devida atenção aos aspectos subjetivos, comuns da adolescência, o que fragiliza a adesão ao tratamento, potencializando comportamentos desfavoráveis. Há a necessidade de uma abordagem ampla para o autocuidado através dos profissionais de saúde, com habilidades de comunicação sobre a condição do adolescente em viver com psoríase, valorizando os aspectos emocionais desta fase para o planejamento do cuidado de si. É importante o alinhamento junto aos jovens e seus familiares, no apoio ao autocuidado ${ }^{(18)}$.

A Organização Mundial de Saúde ${ }^{(29)}$ coloca que a velhice cronologicamente tem início aos 60 anos nos países em desenvolvimento e aos 65 anos nos países desenvolvidos. Mosquera ${ }^{(27)}$ e Dardengo e 
Mafra $^{(30)}$ relatam a complexidade do conceito de velhice, que envolve aspectos biológicos, sociais, psicológicos, culturais, econômicos, entre outros.

Ao desenvolver orientações de autocuidado às pessoas idosas, deve-se ter atenção às limitações que as pessoas deste grupo possam ter. As orientações são dirigidas aos idosos, a seus familiares e cuidadores na realização de tratamentos adequados a esta faixa etária e ao estilo de vida próprio. Neste sentido, enfermeiras comunitárias realizaram orientações de autocuidado ao idoso com psoríase voltadas para o cuidado e preservação da pele, considerando a fragilidade da pele do idoso e as alterações provenientes da psoríase. $\mathrm{O}$ uso de medicação tópica, emolientes e hidratantes foram orientados quanto à escolha, modo de uso e horários. Aconselhamentos para evitar 'gatilhos' desencadeadores da doença; busca de grupos de apoio; adaptações para maior autonomia na realização do autocuidado foram realizados, respeitando as limitações impostas pela idade, grau de autonomia e desejos dos pacientes ${ }^{(17)}$.

Em concordância, Batista e Crepaldi $^{(31)}$ descrevem que o manejo com paciente idoso deve ser abrangente, considerando as peculiaridades da idade, os aspectos sociais e econômicos, grau de autonomia, comorbidades, qualidade de vida existente, habilidade para a realização do autocuidado, além de ter atenção a situação do cuidador, quando for o caso, dentre outros https://doi.org/10.31011/reaid-2021-v.95-n.36-art.1201 Rev Enferm Atual In Derme v. 95, n. 36, 2021 e-021185 fatores como expectativas e escolhas do paciente.

\section{Demandas de autocuidado na perspectiva} dos requisitos de autocuidado no desvio da saúde

Os requisitos de autocuidado no desvio da saúde determinam quais as necessidades de, autocuidado que as pessoas precisam diante de doenças, incapacidades e deficiências, com o propósito de recuperação, controle e reabilitação ${ }^{(6)}$.

$\mathrm{Na}$ psoríase os requisitos de autocuidado no desvio da saúde demandam do paciente conhecimentos sobre a doença, opções de tratamento, medicações (seus efeitos, efeitos adversos e uso), sobre as possíveis comorbidades, adoção de estilos de vida (hábitos alimentares e nutricionais, exercícios e atividades físicas, controle do estresse, uso de tabaco e bebidas alcoólicas, outros), requerendo escolhas conscientes. Estes requisitos, estão diretamente relacionados aos problemas de saúde diagnosticados ou identificados na pessoa com psoríase e exige cuidados diversificados, seja pela própria demanda da doença e/ou pelo risco em desenvolver comorbidades e/ou pela situação de estigma e preconceito que seus sinais e sintomas produzem.

Nos estudos em que se propõem a criação e uso de tecnologia digital na propositura do autocuidado através de aplicativos, foi percebida uma maior abrangência de ações. Os aplicativos dispõem 

psoríase, comorbidades e tratamento, além de outros tópicos relacionados ao estilo de vida, e utilização de fármacos. As ações de autocuidado também abrangem a saúde mental do paciente na atenção aos quadros de estresse, depressão e ansiedade. Além do acompanhamento ao uso de medicação para este fim, os aplicativos indicam e orientam práticas como atividade física, yoga, meditação dentre outras técnicas de relaxamento. Outra função dos aplicativos são os lembretes, onde são programados os agendamentos de horários dos medicamentos, com explicações do uso, principalmente das medicações tópicas, como emolientes e pomadas no manejo das lesões psoriásicas cutâneas. O gerenciamento nutricional e de estilo de vida, também são opções de uso nos $\operatorname{aplicativos}^{(15,20)}$.

$$
\text { As práticas de autocuidado }
$$
desenvolvidas por profissionais de saúde também podem seguir diretrizes padronizadas, baseadas em evidências científicas. Dois estudos desta revisão ${ }^{(17,22)}$, desenvolvidos no Reino Unido, tiveram como diretrizes o National Institute For Health and Care Excellence (NICE) $)^{(32)}$, um instituto independente ligado ao Sistema de Saúde Pública da Grã-Bretanha (NHS). Farmacêuticos comunitários utilizaram as diretrizes do NICE no que tange a atender as necessidades individuais de tratamento do paciente, oferecendo suporte e informações sobre os conhecimentos a respeito da

sobre como usar, de forma eficaz e segura, o tratamento prescrito ${ }^{(22)}$.

No atendimento a idosos com psoríase, enfermeiras comunitárias utilizaram as diretrizes do NICE para os cuidados com a pele e couro cabeludo com uso de emolientes em conjunto com tratamentos tópicos, para melhora do prurido, descamação, fissuras além da hidratação da pele. O NICE também foi usado como diretriz para esclarecimento de diagnóstico e escolha de tratamento, riscos em relação ao estilo de vida, orientações para consultas com especialistas e na discussão de estratégias para lidar com os impactos da doença no bem-estar físico, psicológico e social do paciente ${ }^{(17)}$.

Saber sobre os mecanismos que envolvem a psoríase, sua natureza recorrenteremitente, sua base sistêmica, como ocorre a formação de placas descamativas e as ligações inflamatórias, permitem ao paciente entender a complexidade da doença. Compreender a psoríase como uma doença de longo prazo e desmistificar algumas crenças, permite $o$ paciente a se autocuidar e fazer escolhas de tratamento de forma consciente ${ }^{(14-16,18,20-22)}$.

As opções de tratamento devem ser esclarecidas para o paciente e, com os profissionais de saúde, decidam qual tipo de tratamento melhor atende as necessidades do paciente, incorporando-o em sua rotina. Para tanto, o paciente precisa receber suporte, ser bem orientado e esclarecer suas dúvidas. $\mathrm{O}$ tratamento bem feito, centrado nas necessidades do paciente, contribui https://doi.org/10.31011/reaid-2021-v.95-n.36-art.1201 Rev Enferm Atual In Derme v. 95, n. 36, 2021 e-021185 
positivamente na remissão da doença, adesão ao tratamento e na qualidade de $\operatorname{vida}^{(14,15,17,20,22,33)}$.

As comorbidades se caracterizam como doenças crônicas e devem ser tratadas em sincronia com a psoríase, evitando conflitos entre os tratamentos. É necessário que o paciente conheça a possibilidade do surgimento de algumas comorbidades e se envolva em práticas de autocuidado voltadas para o estilo de vida, como forma preventiva. Quando a doença comorbida for evidenciada, podendo ser uma ou mais, o paciente necessitará ter conhecimento sobre a doença(s) e seu tratamento, recebendo orientações para o autocuidado. Cabe ressaltar que as condições emocionais desencadeadas pela presença de comorbidades, também necessitará do apoio dos profissionais de saúde ${ }^{(14,15,20,21)}$.

A psoríase, assim como toda doença crônica, envolve modificações no estilo de vida e no cotidiano, como também mudanças e/ou aquisição de novos hábitos e rotinas. Neste sentido, exercícios e atividades físicas, técnicas de relaxamento no combate ao estresse, hábitos alimentares favoráveis à saúde e cuidado com a saúde mental são necessários, enquanto práticas de autocuidado $^{(15,17,19,20)}$.

Orientações para a prevenção de 'gatilhos' se apresentam como práticas de autocuidado e abrangem diferentes aspectos tais como cuidados com o uso de abusivo de bebidas alcoólicas e controle do estresse, devendo ter a atenção dos profissionais de saúde ${ }^{(17)}$.

O uso de medicações contraindicadas e/ou inapropriadas deve ser evitado. Medicações a exemplo do lítio, antimaláricos, betabloqueadores dentre outros acabam exacerbando a doença, trazendo prejuízos ao paciente $^{(17)}$.

Para Cassia $^{(34)}$ o tabagismo e etilismo são comportamento de risco contra a integridade física e a saúde. Apesar de estarem associados de maneira independente à gravidade da psoríase, ambos têm impacto negativo em relação à evolução da doença, tanto pelas manifestações inflamatórias quanto pelos fenômenos imunológicos. Corroborando, Fernandes et al. ${ }^{(24)}$ relatam que o tabagismo favorece em $70 \%$ o desencadeamento da psoríase, em comparação às pessoas não fumantes e está associado às diferentes formas de manifestação da psoríase, sendo a pustulosa a mais frequente. Já o alcoolismo contribui para a prevalência da psoríase em pessoas que fazem o uso abusivo, propiciando o maior risco de esteatose alcoólica, baixa resposta aos tratamentos, quadros de depressão e ansiedade e agravamento da doença.

As interações sociais do paciente com psoríase se mostram importantes nos desdobramentos para as práticas do autocuidado. São interfaces das interações sociais as interações interpessoais, os grupos 
de apoio e as relações dos profissionais de saúde com os pacientes ${ }^{(15-20)}$.

As dificuldades nas interações interpessoais produzem comportamentos de isolamento social, consequência do estigma e preconceito vividos no dia-a-dia do paciente. Para não se exporem a críticas e julgamentos, eles acabam evitando exposição pública deixando de realizar diferentes tipos de atividades como exercícios e atividades físicas, atividades de lazer, outras. Tais fatores podem levar os pacientes a adotarem estilo de vida prejudicial à saúde, a exemplo do uso abusivo de álcool e sedentarismo. Neste sentido, as orientações de autocuidado se voltarão para práticas psicossociais que os apoiem no incentivo da participação $\operatorname{social}^{(18,19)}$.

As ações de autocuidado que promovem abordagens de grupo, presenciais ou virtuais, fomentam um senso de comunidade sendo identificados como mecanismos importantes para aprender sobre as condições da doença e o autocuidado. Nestes espaços, as trocas de experiências são comuns. Informações e estratégias de enfrentamento servem de apoio e minimização do sentimento de solidão ${ }^{(16-18)}$.

A importância da interação entre os profissionais de saúde e pacientes reflete na formação de vínculo e confiança. Destaca-se que quando os profissionais de saúde buscam uma melhor comunicação com o paciente, a partir do entendimento sobre seu cotidiano e o apoiado, seguro e acolhido em suas necessidades, favorecendo seu protagonismo no tratamento e gerenciamento do cuidado de $\mathrm{si}^{(14,15-20)}$.

\section{CONSIDERAÇÕES FINAIS}

$\mathrm{O}$ estudo possibilitou identificar e analisar evidências científicas acerca das ações de autocuidado desenvolvidas por profissionais de saúde às pessoas com psoríase dos últimos 5 anos. A análise foi realizada na perspectiva da Teoria do Déficit de Autocuidado, o que possibilitou formular as categorias para melhor compreensão.

Os achados apontam que para o desenvolvimento das ações de autocuidado é necessário identificar quais os requisitos de autocuidado do paciente precisam ser atendidos, optar pela metodologia mais adequada a ser adotada e planejar as ações conforme as necessidades dos pacientes, podendo ou não seguir diretrizes padronizadas. Para tanto, a educação em saúde se coloca como essencial na comunicação dessas práticas, através de estratégias, utilizando-se de diferentes tecnologias em saúde.

$\mathrm{Na}$ atenção ao paciente com psoríase as ações de autocuidado objetivam atender os déficits de autocuidado identificados em cada requisito de autocuidado. Em relação aos requisitos universais, as ações de autocuidado identificadas no estudo foram referentes a dieta alimentar e nutricional, ingestão de 
líquido, atividades físicas e descanso além de ações voltadas as dimensões psicossociais.

No que tange aos requisitos de desvio da saúde várias ações foram identificadas. Informações e orientações sobre o conhecimento da doença, opções de tratamento e conhecimento das medicações, seus efeitos e o uso apropriado; cuidados com a pele e couro cabeludo; identificação dos 'gatilhos' para evitar a exacerbação da doença; orientações e esclarecimento sobre estilo de vida e hábitos saudáveis. Outra ação identificada no estudo foi de orientações para os pacientes ativarem os serviços da rede de saúde em busca de atendimentos especializados, medicações e outros. O incentivo a participação dos pacientes em grupos de autocuidado e associações de apoio a psoríase também fazem parte das práticas de autocuidado realizadas pelos profissionais de saúde.

As condições cognitivas, psicológicas, motoras e sociais de cada fase do desenvolvimento humano em que se encontra o paciente, devem ser consideradas pelo profissional de saúde ao identificar os déficits de autocuidado e planejar as estratégias adequadas de modo a sanar estes déficits, atendendo às especificidades individuais do paciente. Estas condições reverberam na participação social e atividades cotidianas, lazer, trabalho, dentre outras.

Os estudos apontam a potencialidade das ações de autocuidado em psoríase, chamando a atenção dos profissionais de https://doi.org/10.31011/reaid-2021-v.95-n.36-art.1201 Rev Enferm Atual In Derme v. 95, n. 36, 2021 e-021185 saúde para além da clínica medicamentosa. Espera-se que seja fonte de motivação para novos estudos, principalmente as latinoamericanas, dentre esses os brasileiros.

\section{REFERÊNCIAS}

1. Accioly Filho JW. Fatores etiológicos, de risco, desencadeantes e agravantes na psoríase. In: Carneiro SCDAS, Silva MRE, editors. Fundamentos de Psoríase. 1a edição. Rio de Janeiro RJ: Atheneu; 2018. p. 37 - 42.

2. Carneiro SCDAS, Silva MRE. Introdução a psoríase. In: Carneiro SCDAS, Silva MRE, editors. Fundamentos de Psoríase. 1a edição. Rio de Janeiro - RJ: Atheneu; 2018. p. $03-06$.

3. Ortigosa LCM, Lima X. Psoríase em placas: quadro clínico e diagnóstico. In: PALMA S, editor. Consenso brasileiro de psoríase 2020: algoritmo de tratamento da Sociedade Brasileira de Dermatologia. 3a edição. Rio de Janeiro: Sociedade Brasileira de Dermatologia; 2020. p. 25-7.

4. Reichert-Faria A., Castro CCS. Prevalências de comorbidades. In: Palma S, editor. Consenso brasileiro de psoríase 2020: algoritmo de tratamento da Sociedade Brasileira de Dermatologia. 3a edição. Rio de Janeiro: Sociedade Brasileira de Dermatologia; 2020. p. 22-4.

5. Scaccabarozzi L, Del Rey C, Kim H, Amaral LM, Fernandes RA, Asano E, et al. Análise de custo por resposta dos medicamentos biológicos no tratamento da psoríase moderada a grave sob as perspectivas dos sistemas de saúde público e privado, no Brasil. J bras econ saúde. 2016;155-63.

6. Orem DE, Taylor SG, Renpenning KM. Nursing: Concepts of practice. 
1995

7. Silva JV da, Haddad JG V, Pereira MIM, Lima RS. Teoria de enfermagem do Déficit do autocuidado-Dorothea Orem. In: Silva JV., Braga CG, editors. Teorias de enfermagem. 1a edição. São Paulo: ed. Érica; 2011. p. 85-103.

8. Prado Solar LA, González Reguera M, Paz Gómez N, Romero Borges K. La teoría Déficit de autocuidado: Dorothea Orem punto de partida para calidad en la atención. Rev médica electrónica. 2014;36(6):835-45.

9. Hernández YN, Pacheco JAC, Larreynaga MR. La teoría déficit de autocuidado: Dorothea Elizabeth Orem. Gac médica espirituana. 2017;19(3).

10. Ganong LH. Integrative reviews of nursing research. Res Nurs Health. 1987;10(1):1-11.

11. Polit DF, Beck CT. Fundamentos de pesquisa em enfermagem: avaliação de evidências para a prática da enfermagem. Artmed Editora; 2011.

12. Page MJ, McKenzie JE, Bossuyt PM, Boutron I, Hoffmann TC, Mulrow CD, et al. The PRISMA 2020 statement: an updated guideline for reporting systematic reviews. Bmj. 2021;372.

13. Loreiro MCAC, Carneiro S. Epidemiologia. In: Carneiro S., Ramose-Silva., editors. Fundamentos de Psoríase. 1a edição. Rio de Janeiro RJ: Atheneu; 2018. p. 29-33.

14. Larsen MH, Strumse YAS, Borge CR, Osborne R, Andersen MH, Wahl AK. Health literacy: a new piece of the puzzle in psoriasis care? A cross-sectional study. Br J Dermatol. 2019;180(6):1506-16.

15. Zhu B, Wang Y, Zhou X, Cao C, Zong $\mathrm{Y}$, Zhao X, et al. A controlled study of the feasibility and efficacy of a cloud- based interactive management program between patients with psoriasis and physicians. Med Sci Monit Int Med J Exp Clin Res. 2019;25:970.

16. Larsen MH, Strumse YS, Andersen MH, Borge CR, Wahl AK. Associations between disease education, self-management support, and health literacy in psoriasis. $\mathbf{J}$ Dermatolog Treat. 2019;1-7.

17. Onselen J Van. Self-care for older people with psoriasis. Br J Community Nurs. 2018;23(5):214-7.

18. Rasmussen GS, Kragballe K, Maindal HT, Lomborg K. Experience of being young with psoriasis: self-management support needs. Qual Health Res. 2018;28(1):73-86.

19. Khoury LR, Skov L, Møller T. Facing the dilemma of patient-centred psoriasis care: a qualitative study identifying patient needs in dermatological outpatient clinics. Br J Dermatol. 2017;177(2):436-44.

20. Safdari R, Firoz A, Masoorian H. Identifying training and informational components to develop a psoriasis selfmanagement application. Med J Islam Repub Iran. 2017;31:67.

21. Nelson PA, Kane K, Pearce CJ, Bundy C, Chisholm A, Hilton R, et al. 'New to me': changing patient understanding of psoriasis and identifying mechanisms of change. The Pso Well ${ }^{\circledR}$ patient materials mixed-methods feasibility study. $\mathrm{Br} \mathrm{J}$ Dermatol. 2017;177(3):758-70.

22. Tucker R, Stewart D. The role of community pharmacists in supporting self-management in patients with psoriasis. Int $\mathbf{J}$ Pharm Pract. 2017;25(2):140-6.

23. Oliveira M de FSP de, Rocha B de O, Duarte GV. Psoriasis: classical and emerging comorbidities. An Bras https://doi.org/10.31011/reaid-2021-v.95-n.36-art.1201 Rev Enferm Atual In Derme v. 95, n. 36, 2021 e-021185 
Dermatol. 2015;90:9-20.

24. Fernandes B, Lima JM, Azevedo MEH de, Bulhões SA de, Costa JEF da. Comorbidades e multidisciplinaridade na doença psoriásica. In: Soares VL, Palitot EB, Callou KR de A, editors. Da ciência ao cuidado: saberes e práticas em psoríase. 1a edição. João Pessoa: Universidade Federal da Paraíba (UFPB); 2021. p. 116-28.

25. Melo SP da S de C, Cesse EÂP, Lira PIC, Rissin A, Cruz R de SBLC, Batista Filho M. Doenças crônicas não transmissíveis e fatores associados em adultos numa área urbana de pobreza do nordeste brasileiro. Cien Saude Colet. 2019;24:3159-68.

26. Cunha GH da, Ramalho AKL, Cruz AMM, Lima MAC, Franco KB, Lima RCR de O. Diagnósticos de enfermagem segundo a teoria do autocuidado em pacientes com infarto do miocárdio. 2018.

27. Mosquera JJM. Vida adulta: personalidade e desenvolvimento. 2a edição. Porto Alegre: Livraria Sulina Editora; 1983.

28. Soares VL, Blascovi-Assis SM. Influência do esporte adaptado na autoimagem do adolescente com deficiência física. In: Galvão CRC., Polia AA., Gomes MQC, editors. Olhares e perspectivas da Terapia Ocupacional: construindo teorias e pensando a prática. João Pessoa: Universidade Federal da Paraíba (UFPB); 2021. p. 111-21.

29. Organização Mundial da Saúde. Envelhecimento ativo: uma política de saúde. 2005;

30. Dardengo CFR, Mafra SCT. Os conceitos de velhice e envelhecimento ao longo do tempo: contradição ou adaptação? Rev Ciências Humanas. 2018;(2).
31. Batista EFVM., Crepaldi N. Idosos. In: Carneiro S., Ramos-e-Silva., editors. Fundamentos de Psoríase. 1a edição. Rio de Janeiro - RJ: Atheneu; 2018. p. 159-62.

32. National institute for health and care excellence. NICE guidance [Internet]. 2021. Available from: https://www.nice.org.uk/guidance

33. Thorneloe RJ, Griffiths CEM, Emsley $\mathrm{R}$, Ashcroft DM, Cordingley L, Barker $\mathrm{J}$, et al. Intentional and unintentional medication non-adherence in psoriasis: the role of patients' medication beliefs and habit strength. J Invest Dermatol. 2018;138(4):785-94.

34. Cassia FF. Tabagismo e alcoolismo. Epidemiologia. In: Carneiro S., Ramose-Silva., editors. Fundamentos de Psoríase. 1 ed. Rio de Janeiro - RJ: Atheneu; 2018. p. 237-238.

\section{Autor correspondente}

Valéria Leite Soares; Rua Ana Cristina Rolim Machado, 264, ap. 107, Aeroclube, João PessoaParaíba, CEP 58.036-444. Cel. +55

(83)999929424. E-mail:

valeriasoares1@hotmail.com

Submissão: 2021-08-16

Aprovado: 2021-11-04 